\title{
Farnesyl Protein Transferase Inhibition Interferes with Activation of MAP Kinase Family Members in Human Peripheral Blood Monocytes
}

\author{
Walter L. Vervenne, ${ }^{1}$ Carina L. Bos, ${ }^{2}$ Linda S. Rens, ${ }^{2}$ Maikel P. Peppelenbosch, ${ }^{2}$ and Dick J. Richel ${ }^{1}$ \\ ${ }^{1}$ Department of Medical Oncology \\ ${ }^{2}$ Laboratory for Experimental Internal Medicine, Academic Medical Center, Amsterdam, The Netherlands \\ Accepted October 14, 2002
}

\begin{abstract}
Background: Farnesyl protein transferase inhibitors have emerged as promising novel agents for combating cancerous disease. Nevertheless, the importance for farnesyl protein transferase enzymatic activity for cellular physiology of untransformed cells remains poorly investigated.

Materials and Methods: Peripheral blood monocytes, isolated from the blood of eight healthy volunteers, were treated with a farnesyl protein transferase inhibitor (FTI 744,832) or vehicle control for $16 \mathrm{hr}$. Subsequently cells were challenged with different concentrations of lipopolysaccharide (LPS), colony stimulating factor-1 (CSF-1), or phorbol esters for $10 \mathrm{~min}$, after which the activation state of p42/p44 MAP kinase, p38 MAP kinase, and Jun-N-terminal kinase was investigated using Western blotting and phosphospecific antibodies.
\end{abstract}

Results: We observed that farnesyl protein transferase inhibition abrogated activation of p38 MAP kinase by LPS, CSF-1, and phorbol esters. Also the activation of Jun-Nterminal kinase by LPS was not seen after farnesyl protein transferase inhibition. Finally, stimulation of p42/p44 MAP kinase with CSF-1 was strongly reduced by farnesyl protein transferase inhibition, whereas activation of $\mathrm{p} 42 / \mathrm{p} 44$ MAP kinase by phorbol ester was only slightly effected.

Conclusions: Farnesyl protein transferase enzymatic activity is required for proper activation of all major members of the MAP kinase family. The observation that activation the p38 MAP kinase and Jun- $\mathrm{N}$-terminal kinase is sensitive to farnesyl protein transferase inhibition raises the possibility that, in addition to cancerous disease, farnesyl protein transferase inhibitors may be useful compounds in combating inflammatory disease.

\section{Introduction}

Farnesyl protein transferase transfers farnesyl isoprenoid (which is an intermediate in cholesterol biosynthesis) to polypeptide chains and thus is required for the biological activity of a variety of proteins. In particular, various members of the Ras family of small GTPases require posttranscriptional farnesylation for biological function and thus farnesyl protein transferase inhibitors (FTI) have been suggested as a novel class of anti-tumor agents. In accordance, FTIs suppress the growth of Ras-dependent tumors (1) and a large body of preclinical data obtained in both in vitro and in vivo has provided support for the potential therapeutic usefulness of these compounds (2). FTIs have been shown to block and even reverse Ras-induced transformation, to inhibit the growth of many cancer cell lines in vitro (3-7), and to impair proliferation of Rasactivated xenografts in nude mice (see, for example, Sun et al. [8]). In addition, farnesyl protein transferase interferes with the biological function of

W.L.V. and C.L.B. contributed equally to this manuscript. Address correspondence and reprint requests to: Maikel P. Peppelenbosch, Laboratory for Experimental Internal Medicine, G2-130, Academic Medical Center, Meibergdreef 9, 1105 AZ Amsterdam, The Netherlands. Phone: (31)-20-5665955; fax: (31)-20-6919743; e-mail: M.P.Peppelenbosch@amc.uva.nl
RhoB. This small GTPase, uniquely, exists in both a farnesylated and a geranylgeranylated form (9). FTItreated cells will quickly display decreased levels of farnesylated RhoB, whereas geranylgeranylated RhoB levels will be enhanced. RhoB may be genetically engineered to allow only geranylgeranylation and no farnesylation. When such forms of RhoB are overexpressed in Ras-transformed cells, phenotypic reversion, cell growth inhibition, and induction of the cell cycle inhibitor p2IWAF is observed. Thus, elevating geranylgeranylated RhoB levels in cells induce effects which closely resemble those of FTIs (10) and RhoB is a crucial target for alteration by FTIs and its relevance to anti-oncogenic therapy has gained significant acceptance.

Nevertheless, the consequences of farnesyl protein transferase inhibition for cellular physiology remain largely poorly understood. Especially the action of farnesyl protein transferase in the cellular physiology of untransformed cells remains obscure. This prompted us to investigate the effect of farnesyl protein transferase inhibition on the activation state of MAP kinases in human peripheral blood monocytes. There is now broad consensus that the MAP kinase family is a major regulator of cell physiology, p42/p44 MAP kinase being associated with cell proliferation; p38 MAP kinase and JNK are associated with inflammatory responses and cell stress, 
respectively. Thus, by investigating the effects of FTI on these three different MAP kinases, we hoped to obtain a fairly comprehensive overview of the role of farnesyl protein transferase in normal cellular physiology. For this purpose we employed FTI L-744,832, a peptidomimetic and very well-characterized farnelsyl:protein transferase inhibitor having an IC50 for human farnesyl protein transferase of approximately $10 \mu \mathrm{M}$ for Ras prenylation in both transformed and untransformed hematopoietic cells, that has been extensively described and of which the specificity has not been questioned (11-15). In the current study, we observed that farnesyl protein transferase inhibition abrogated activation of p38 MAP kinase by lipopolysaccharide (LPS), colony stimulating factor-1 (CSF-1), and 12-O-tetradecanoylphorbol 13-acetate (TPA) Also the activation of JNK by LPS was not seen after farnesyl protein transferase inhibition. Finally, stimulation of p42/p44 MAP kinase by CSF-1 was strongly reduced by farnesyl protein transferase inhibition, whereas activation of $\mathrm{p} 42 / \mathrm{p} 44$ MAP kinase by TPA was only slightly effected. Thus, farnesyl protein transferase enzymatic activity is required for proper activation of all major members of the MAP kinase family. The observation that activation the p38 MAP kinase and Jun-N-terminal kinase is sensitive to farnesyl protein transferase inhibition raises the possibility that, in addition to cancerous disease, farnesyl protein transferase inhibitors may be useful compounds in combating inflammatory disease.

\section{Methods}

\section{Cell Preparation and Culture}

Venous blood was drawn from eight healthy volunteers and heparinized with $10 \mathrm{U} / \mathrm{ml}$ heparin (Leo Pharmaceutical Products, Weesp, The Netherlands) and diluted 1:1 with phosphate-buffered saline (PBS) PBMCs were isolated by density-gradient sedimentation on Ficoll-Paque (Pharmacia, Uppsala, Sweden). PBMCs were washed twice with PBS and resuspended in DMEM cell culture medium. Cells were then plated in 6-well plates (Corning Inc. Costar ${ }^{\circledR}$, Corning, NY, USA). After $2 \mathrm{hr}$ medium was removed and one-half of the adherent cells were incubated overnight with FTI 744,832 (Biomol Research Laboratories, Inc., Plymouth Meeting, PA, USA) in a concentration of $20 \mu \mathrm{g} / \mathrm{l}$, with the other half acting as a control. Visual inspection of the cells did not reveal any apparent toxicity after overnight incubation with FTI 744,832. Subsequently, cells were stimulated with TPA, LPS, or CSF-1 at the indicated concentrations for $10 \mathrm{~min}$. Stimulations were terminated by the addition of $5 \mathrm{ml}$ ice-cold PBS. After removing the PBS, cells were lysed in $100 \mu \mathrm{l}$ protein sample buffer (125 mM Tris/HCL, pH 6,8; 4\% SDS, $2 \% \beta$-mercapto-ethanol; $20 \%$ glycerol, $1 \mathrm{mg}$ bromophenol blue), and stored at $-20^{\circ} \mathrm{C}$ until further processing.

\section{Immunoblotting}

Samples were subjected to $12.5 \%$ SDS-PAGE and blotted on to a PVDF membrane (Millipore, Bedford, MA, USA). To check for equal loading of protein in each lane, Coomassie brilliant blue staining of the PVDF membrane was performed. Subsequently, membranes were blocked in $2 \%$ protifar (Nutricia, Zoetermeer, Netherlands) in TBST supplemented with $0.1 \%$ Tween 20 for $1 \mathrm{hr}$ at room temperature. After a brief wash in washing buffer $(0.2 \%$ protifar; $0.1 \%$ Tween 20), membranes were incubated overnight at $4^{\circ} \mathrm{C}$ with antibody (see below) diluted 1:1000 in washing buffer. The following day membranes were washed three times for $5 \mathrm{~min}$, and subsequently incubated with a secondary horseradish peroxidase (HRP)-conjugated antibody in a 1:1000 dilution. After enhanced chemoluminescence using Lumilight substrate (Roche, Mannheim, Germany), antibody binding was visualized using a lumi-imager (Syngene Bio-imaging, Synoptics Ltd., Cambridge, UK.) Equal loading was always verified using $\beta$-actin staining.

\section{Antibodies}

Antibodies used to detect phosphorylated protein were rabbit polyclonal antibodies against phosphorylated p38 MAP kinase (Thr-180/Tyr-182), p42/44 MAP kinase (Thr-202/Tyr-204), Jun-N-terminal kinase (Thr-183/Tyr-185), all from New England Biolabs (Beverly, MA, USA). The secondary antibody used was a HRP-conjugated goat anti-rabbit polyclonal antibody from DAKO (Glostrup, Denmark).

\section{Quantification of Lumi-imager Data}

Phosphorylation of MAPKs was measured by Western blot. Antibody binding was quantified using image analysis software (GeneGenome, Syngene Bioimaging, Synoptics Ltd.) All laboratory-based values are given as means \pm SEM.

\section{ELISA}

For cytokine production, cells were stimulated with various concentrations of LPS for $24 \mathrm{hr}$ as indicated and supernatant was collected. Subsequently tumor necrosis factor (TNF), interleukin-6 (IL-6), and interleukin-8 (IL-8), ELISA was performed according to routine procedures (16).

\section{Results \\ Inhibition of p42/p44 MAP Kinase Activation by FTI $L-744,832$}

To characterize farnesyl protein transferase enzymatic activity on the cellular physiology of untransformed cells, blood was obtained from eight healthy volunteers and peripheral blood human monocytes were isolated. The cells were either exposed to overnight FTI L-744,832 treatment or left untreated 
$\mathbf{A}$

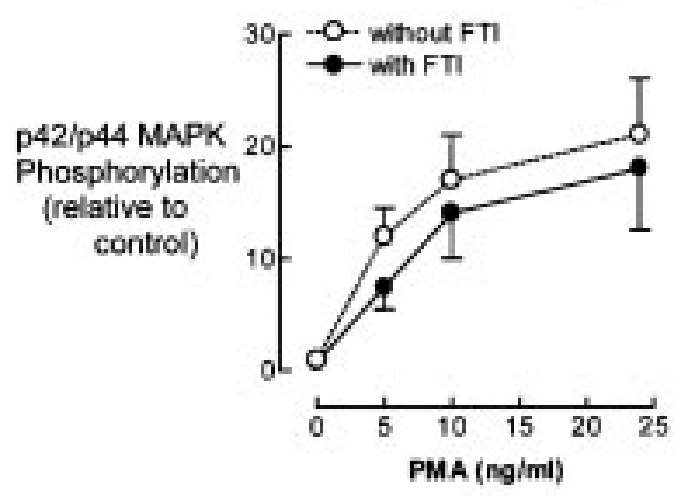

C
B

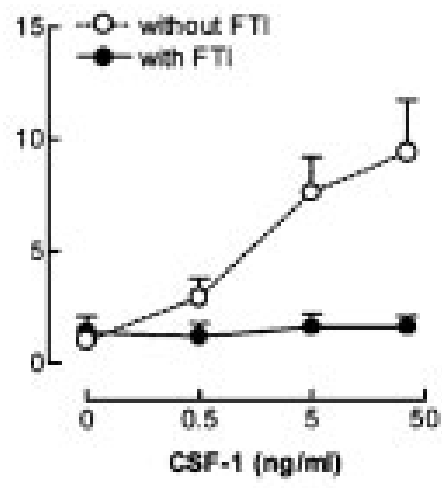

Fig. 1. Effects of farnesyl protein transferase inhibition on $\mathrm{p} 42 / \mathrm{p} 44$ MAP kinase activation. FTIs inhibit phosphorylation of p42/44 MAP kinase after CSF-1 but not after TPA stimulation in human peripheral blood monocytes. Cells were either incubated or not incubated with FTI $(20 \mu \mathrm{M})$ for $16 \mathrm{hr}$ and stimulated afterward with CSF-1 or TPA for $10 \mathrm{~min}$. Phosphorylation of MAPKs was measured by Western blot in eight volunteers. Antibody binding was quantified using image analysis software. All laboratory based values are given as means \pm SEM. (A) Effects of FTI on TPA-induced p42/44 MAP kinase phosphorylation. (B) Effects of FTI on CSF1-induced p42/44 MAP kinase phosphorylation. (C) A representative example of a Western blot analysis of phosphorylated p42/44 MAP kinase after CSF-1 stimulations (increasing concentrations in $\mathrm{ng} / \mathrm{ml}$ ) in absence and presence of FTI. and subsequently stimulated with increasing concentrations of either TPA, CSF-1, or LPS for $10 \mathrm{~min}$, after which cells were harvested and analyzed with Western blotting and phosphospecific antibodies for the activation for MAP kinase family members. We first studied the activation of p42/p44 MAP kinase, which is a principal regulator a variety of biological processes, in particular those linked to mitogenic signaling. We observed concentration-dependent phosphorylation and thus activation of $\mathrm{p} 42 / \mathrm{p} 44$ MAP kinase in response to both TPA and CSF-1, but not to LPS (Fig. 1). Upon inhibition of farnesyl protein transferase, this response was almost completely abolished in CSF-1-stimulated cells (Fig. 1). In contrast, TPA-stimulated cells displayed only slight inhibition of $\mathrm{p} 42 / \mathrm{p} 44$ phosphorylation after farnesyl protein transferase inhibition, demonstrating that the farnesyl protein transferase inhibition effect is not due to cell death or an other generalized toxic effect on cell physiology, but reflects a specific effect on the wiring of cellular signal transduction events. In agreement, farnesyl protein transferase inhibition did not effect phosphorylation of protein kinase $\mathrm{C}$ or actin protein levels in the cells (data not shown) Thus, farnesyl protein transferase inhibition interferes with some but not all signal transduction pathways producing activation of $\mathrm{p} 42 / \mathrm{p} 44$ MAP kinase pathway in untransformed cells.

\section{Inhibition of JNK Phosphorylation after} LPS Stimulation by FTI Treatment

In contrast to p42/p44 MAP kinase, activation of Jun-N-terminal kinase is most prominently linked to the cell stress response. In our experimental system, both TPA and CSF-1 did not produce enhanced phosphorylation of this kinase (data not shown), but a clear concentration-dependent induction of Jun-Nterminal kinase phosphorylation was observed after LPS stimulation. Interestingly, this phosphorylation was completely blocked by FTI L-744,832 (Fig. 2) at all LPS concentrations tested. Apparently, farnesyl protein transferase enzymatic activity is required for cells to respond to LPS challenge with activation of Jun-N-terminal kinase. Thus, in primary isolated mononuclear blood cells, farnesyl protein transferase

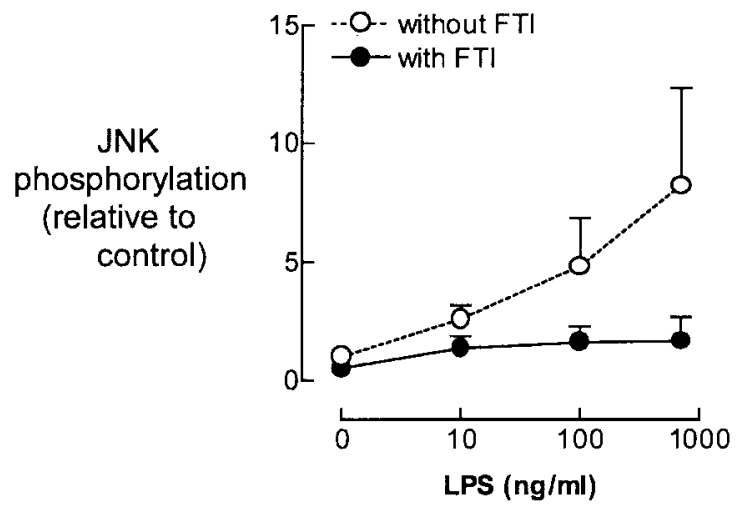

Fig. 2. Effects of farnesyl protein transferase inhibition on JNK activation. FTIs inhibit phosphorylation of JNK after LPS stimulation in human peripheral blood monocytes. Cells were either incubated or not incubated with FTI $(20 \mu \mathrm{M})$ for $16 \mathrm{hr}$ and stimulated afterward with different concentrations of LPS (10 min). Phosphorylation of JNK was measured by Western blot in four volunteers. Antibody binding was quantified using image analysis software. All laboratory based values are given as means \pm SEM. 


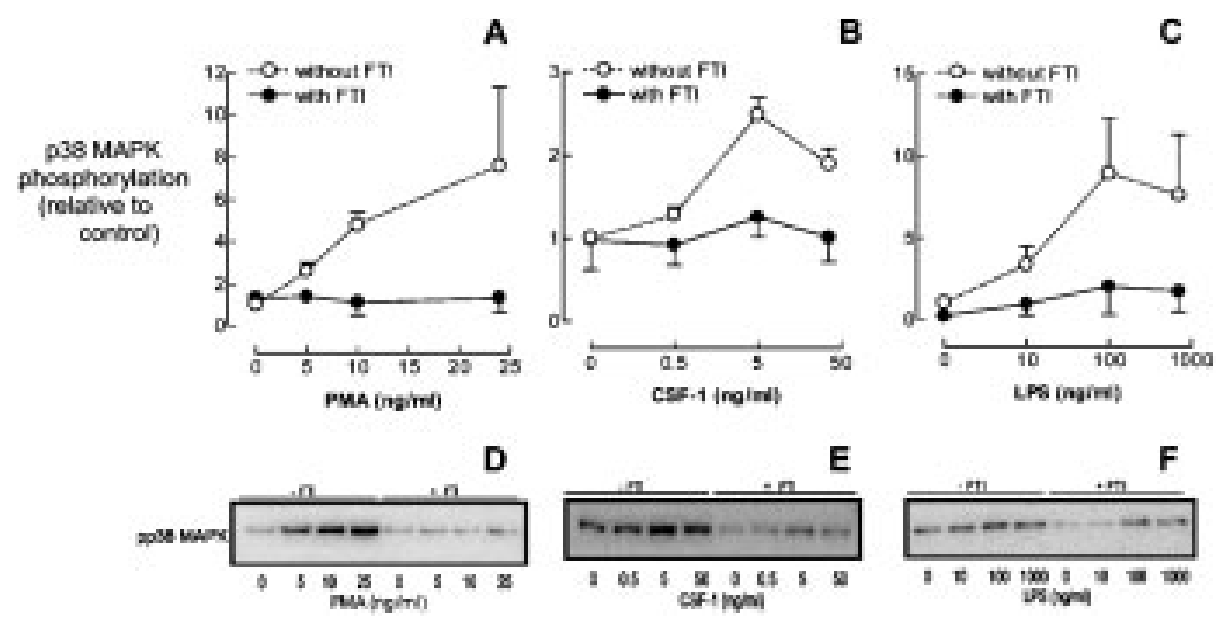

Fig. 3. Effects of farnesyl protein transferase inhibition on p38 MAP kinase activation. FTIs inhibit phosphorylation of p38 MAP kinase after TPA, CSF-1, and LPS stimulation in human peripheral blood monocytes. Cells were either incubated or not incubated with FTI $(20 \mu \mathrm{M})$ for $16 \mathrm{hr}$ and stimulated afterward with TPA, CSF-1 or LPS for 10 min. Phosphorylation of p38 MAP kinase was measured by Western blot in eight volunteers. Antibody binding was quantified using image analysis software. All laboratory based values are given as means \pm SEM. (A) Effects of FTI on TPA-induced p38 MAP kinase phosphorylation. (B) Effects of FTI on CSF-1-induced p38 MAP kinase phosphorylation. (C) Effects of FTI on LPS-induced p38 MAP kinase phosphorylation. (D) A representative example of a Western blot analysis of phosphorylated p38 MAP kinase after TPA stimulation (increasing concentrations in ng/ml) in absence and presence of FTI. (E) A representative example of a Western blot analysis of phosphorylated p38 MAP kinase after CSF-1 stimulation (increasing concentrations in $\mathrm{ng} / \mathrm{ml}$ ) in absence and presence of FTI. (F) A representative example of a Western blot analysis of phosphorylated p38 MAP kinase after LPS stimulation (increasing concentrations in $\mathrm{ng} / \mathrm{ml}$ ) in absence and presence of FTI.

inhibition does not only influence mitogenic signaling associated with $\mathrm{p} 42 / \mathrm{p} 44$ MAP kinase, but also the LPS-induced stress response pathway.

\section{Inhibition of Activation of p38 MAP Kinase by FTI L-744,832 in Three Stimulation Models}

Finally, we tested the effects of farnesyl protein transferase inhibition on the activation of p38 MAP kinase, which is associated with inflammatory responses. Somewhat to our surprise, we detected enhanced phosphorylation of p38 MAP kinase in response to LPS and CSF as well as TPA, and all three stimuli elicited this enhanced phosphorylation in a concentration-dependent manner (Fig. 3). Strikingly, inhibition of farnesyl protein transferase abolished the increased p38 MAP kinase phosphorylation in response to all three different challenges and reduced basal p38 MAP kinase phosphorylation. Therefore, our results show that inhibition of $\mathrm{p} 38$ MAP kinase is a general effect in response to farnesyl protein transferase inhibition. We conclude that in primary isolated human peripheral blood cells the activation of all three major members of the MAP kinase family require functional farnesyl protein transferase.

\section{Functionality of FTI L-744,832 Effects on the LPS Response}

We observed earlier that p38 MAP kinase inhibition increases cytokine release in macrophages (16). To investigate whether a similar effect was noted in FTI-treated PBMCs, we stimulated cells with LPS in presence or absence of FTI. As evident from Figure 4, FTI treatment enhanced TNF but not IL- 6 and IL- 8 release by monocytes. Thus, the inhibition of p38 MAP kinase activity has functional consequences and FTI treatment does not interfere with cell survival per se. The latter notion was also confirmed in experiments in which the effects of FTIs on caspase activity were investigated. FTI treatment did not influence caspase 3 levels in these cells, and did also not interfere with the LPSinduced reduction in caspase 3 activation. Thus, farnesyl protein transferase activity seems not involved in LPS-induced survival signaling in these cells, but apparently is also not cytotoxic.

\section{Discussion}

FTIs attract widespread interest as a consequence of their outstanding anti-tumor efficacy and minimal toxicity in preclinical models of cancerous disease. The importance of farnesyl protein transferase for normal cellular physiology remains, however, unclear. This consideration prompted us to investigate the effect of farnesyl protein transferase inhibition on the activation of the major members of the MAP kinase family in human peripheral blood monocytes. Our results show that activation of $\mathrm{p} 42 / \mathrm{p} 44$ MAP kinase, p38 MAP kinase, and Jun-N-terminal kinase is influenced by FTI treatment. Thus, farnesyl protein transferase enzymatic activity seems essential for a wider range of biological processes than was thought hitherto. A trivial explanation of these results may be that the effects observed reflect a general effect on cell survival rather than a specific 
A

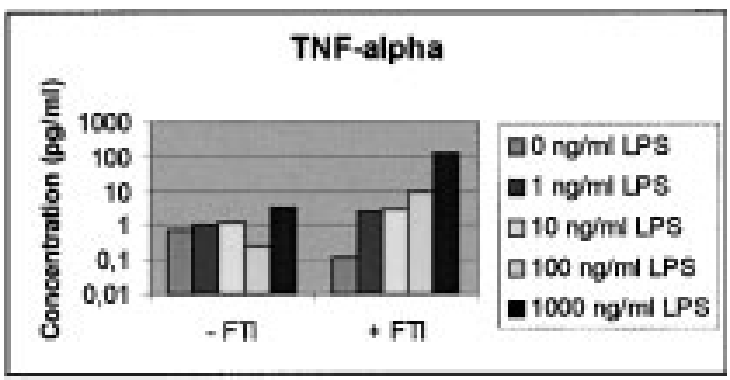

B

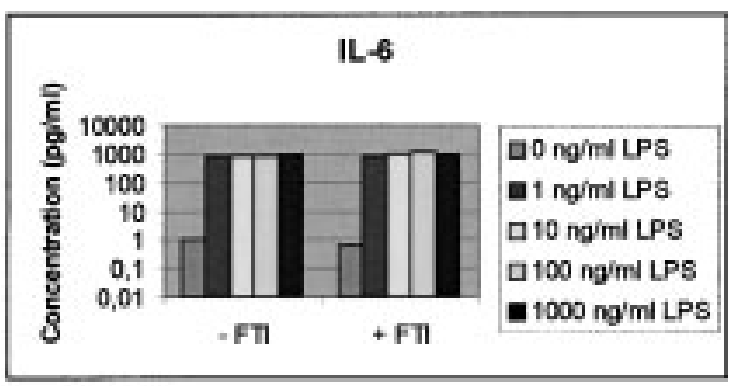

C

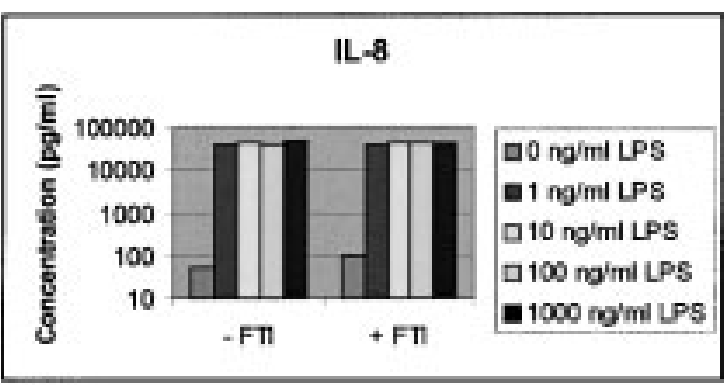

Fig. 4. FTIs influence cytokine release. Cells were stimulated with LPS or $24 \mathrm{hr}$ in the presence or absence of FTIs. Supernatants were collected and cytokine levels determined. (A) TNF levels. (B) IL-6 levels. (C) IL-8 levels.

effect of farnesyl protein transferase per se. Treatment with FTI, however, did not result in obvious cell toxicity as judged by appearance, cellular protein content, the activation of protein kinase $\mathrm{C}$, or the phorbolester-dependent stimulation of $\mathrm{p} 42 / \mathrm{p} 44$ MAP kinase. Hence, we assume that effects observed are specific and reflect a genuine broad requirement for functional farnesyl protein transferase for activation of MAP kinase family members in human peripheral blood monocytes.

A requirement for farnesyl protein transferase enzymatic activity for the activation of $\mathrm{p} 42 / \mathrm{p} 44$ MAP kinase, p38 MAP kinase and Jun-N-terminal kinase could be seen as inconsistent with the reported minimal toxicity of FTIs. However, both in animal studies as well as in human studies, inhibitors of p42/p44 MAP kinase (17), or p38 MAP kinase $(16,18)$ showed little toxicity, whereas CNI-1493, a combined inhibitor of both jun-N-terminal kinase and p38 MAP kinase, exhibited little side effect in humans as well (19). Apparently, MAP kinases can be inhibited in vivo without gross physical disturbances. Interestingly, CNI-1493, a combined

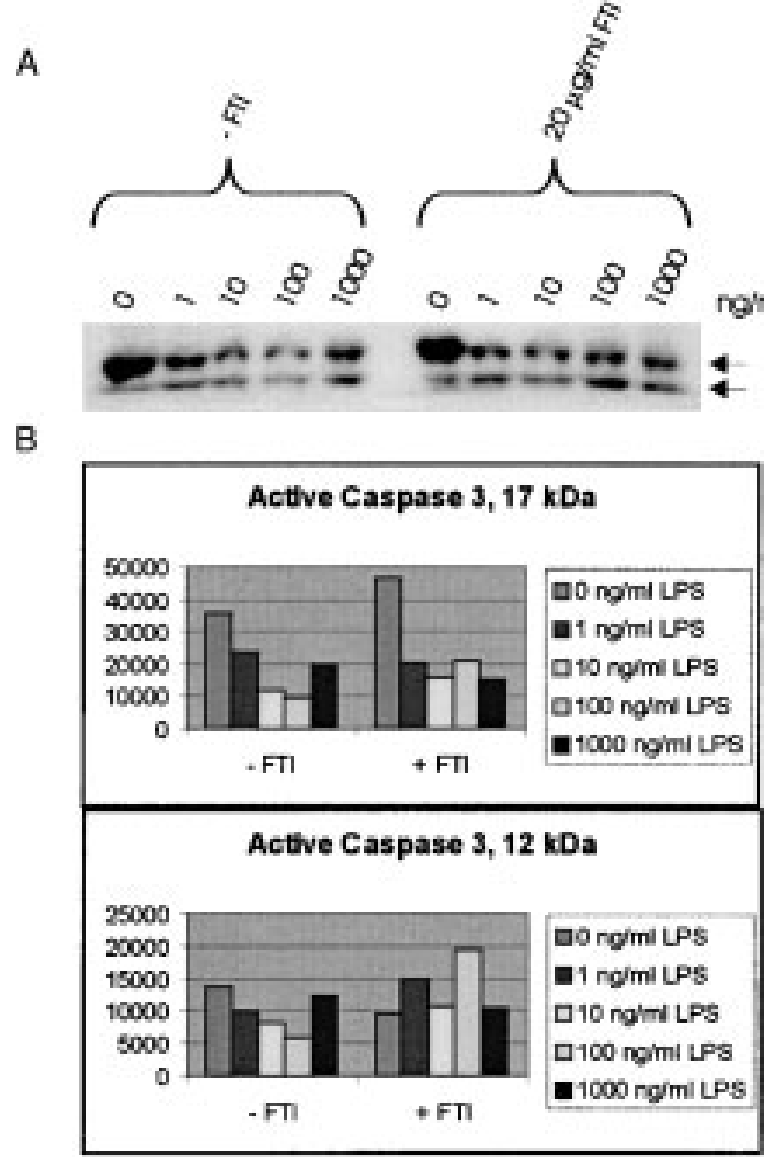

Fig. 5. FTIs do not influence apoptosis. Cells were stimulated for $24 \mathrm{hr}$ in the presence or absence of FTIs and taken up in sample buffer. Using an antibody directed against active caspase 3 and western blotting the effects on LPS induced survival signaling were determined. A. Western blot. B. Quantification.

inhibitor of jun-N-terminal kinase and p38 MAP kinase, was remarkably effective in steroid refractory Crohn's disease (19), whereas the p38 MAP kinase inhibitor BIRB 796 BS is an anti-inflammatory drug in human endotoxemia (18). Thus, our results that FTIs interfere with activation of p38 MAP kinase and Jun-N-terminal kinase indicate that the potential clinical usefulness of FTIs may not be limited to cancerous disease, but that these compounds may also become effective drugs for immunomodulation. This notion is strengthened by our observation that FTIs influence the LPS-induced cytokine production. Importantly, however, the observation that FTIs do not increase apoptosis in nontransformed PBMCs, as judged by caspase 3 activation, strongly support a role of FTIs as anti-oncogenic drugs: inducing apoptosis in malignant cells but not in untransformed cells. The notion that FTIs may have different effects in transformed cells when compared to untransformed cells is further confirmed by the inhibition of JNK activation. Whereas Mizukami et al. (20) see JNK activation in response to FTI in transformed cells, we observed diminished JNK activation in 
FTI-treated untransformed cells. With regard to the therapeutic application of FTIs, it is important to note that the enhanced cytokine production in FTItreated cells might lead to averse events with respect to allergic responses (but may be beneficial in immunocompromised patients). Studies in patients will be necessary to determine which of these partially counteracting effects prevail in vivo.

Classically, FTIs have been shown to block and even reverse Ras-induced transformation and to inhibit the growth of many cancer cell lines in vitro (2-7) and to impair proliferation of RAS-activated xenografts in nude mice (8). Our observation that FTI treatment interferes with p42/p44 MAP kinase stimulation by CSF-1 is in good agreement with such inhibition of Ras. Also, the observation that the TPA-induced stimulation of MAP kinase family members is not markedly sensitive to farnesyl protein transferase inhibition is not inconceivable; it is well established that this phorbol ester acts as a diacylglycerol mimetic, directly activating PKC, which in turn can activate p42/p44 MAP kinase in a Rasindependent fashion via direct phosphorylation of Raf (21). Thus, our data are in agreement with Ras signal transduction being a target for farnesyl protein transferase inhibition, although considering the half-life of Ras and the 16-hr incubation period, there is the possibility for other targets in FTI effects. Indeed, the observation that in our experimental system LPS-induced p38 MAP kinase and JNK phosphorylation is sensitive to farnesyl protein transferase inhibition, but that we were not able to detect p42/p44 MAP kinase phosphorylation, suggests that Ras is not activated by LPS, and thus an alternative target for farnesyl protein transferase inhibition. In agreement, there is little evidence in the literature that Ras is involved in the stimulation of either p38 MAP kinase or JNK, pointing to other possibilities must be considered. RhoB, a low-molecular-weight GTPase that is both farnesylated and geranylgeranylated, is often proposed as a relevant target for FTI's $(10,21,22)$, and may also be so with regard to the effects observed in present study, but evidently further experimental work is required to support this notion. Disregarding the molecular mechanism mediating FTI effects on MAP kinase activation, the present study has shown that in untransformed cells farnesyl protein transferase enzymatic activity is required for functional activation of all three major members of the MAP kinase family, revealing that this enzyme is essential for more events in signal transduction as assumed hitherto.

\section{References}

1. Gibbs JB, Oliff A. (1997) The potential of farnesyltransferase inhibitors as cancer chemotherapeutics. Annu. Rev. Pharmacol. Toxicol. 37: 143-166.

2. Cox AD, Der CJ. (1997) Farnesyltransferase inhibitors and cancer treatment: targeting simply Ras? Biochim. Biophys. Acta 1333: F51-F71.
3. James GL, Goldstein JL, Brown MS, et al. (1993) Benzodiazepine peptidomimetics: potent inhibitors of Ras farnesylation in animal cells. Science 260: 1937-1942.

4. Manne V, Yan N, Carboni JM, et al. (1995) Bisubstrate inhibitors of farnesyltransferase: a novel class of specific inhibitors of ras transformed cells. Oncogene 10: 1763-1779.

5. Nagasu T, Yoshimatsu K, Rowell C, Lewis MD, Garcia AM. (1995) Inhibition of human tumor xenograft growth by treatment with the farnesyl protein transferase inhibitor B956 Cancer Res. 55: 5310-5314.

6. Prendergast GC, Davide JP, deSolms SJ, et al. (1994) Farnesyltransferase inhibition causes morphological reversion of rastransformed cells by a complex mechanism that involves regulation of the actin cytoskeleton NE. Mol. Cell. Biol. 14: 4193-4202.

7. Sepp-Lorenzino L, Ma Z, Rands E, et al. (1995) A peptidomimetic inhibitor of farnesyl:protein transferase blocks the anchorage-dependent and -independent growth of human tumor cell lines. Cancer Res. 55: 5302-5309.

8. Sun J, Qian Y, Hamilton AD, Sebti SM. (1995) Ras CAAX peptidomimetic FTI 276 selectively blocks tumor growth in nude mice of a human lung carcinoma with K-Ras mutation and p53 deletion. Cancer Res. 55: 4243-4247.

9. Adamson P, Marshall CJ, Hall A, Tilbrook PA. (1992) Posttranslational modifications of p2lrho proteins. J. Biol. Chem. 267: 20033-20038.

10. Du W, Lebowitz PF, Prendergast GC. (1999) Cell growth inhibition by farnesyltransferase inhibitors is mediated by gain of geranylgeranylated RhoB. Mol. Cell. Biol. 19: 1831-1840.

11. Sepp-Lorenzino L, Ma Z, Rands E. et al. (1995) A peptidomimetic inhibitor of farnesyl:protein transferase blocks the anchorage-dependent and -independent growth of human tumor cell lines. Cancer Res. 55: 5302-5309.

12. Kohl NE, Omer CA, Conner MW, et al. (1995) Inhibition of farnesyltransferase induces regression of mammary and salivary carcinomas in ras transgenic mice. Nat. Med. 1: 792-797.

13. Norgaard P, Law B, Joseph H, et al. Treatment with farnesylprotein transferase inhibitor induces regression of mammary tumors in transforming growth factor (TGF) alpha and TGF alpha/neu transgenic mice by inhibition of mitogenic activity and induction of apoptosis. Clin. Cancer Res. 5: 35-42.

14. Law BK, Norgaard P, Gnudi L, et al. (1999) Inhibition of DNA synthesis by a farnesyltransferase inhibitor involves inhibition of the p70(s6k) pathway. J. Biol. Chem. 274: 4743-4748.

15. Mahgoub N, Taylor BR, Gratiot M, et al. (1999) In vitro and in vivo effects of a farnesyltransferase inhibitor on $\mathrm{Nf1-}$ deficient hematopoietic cells. Blood 94: 2469-2476.

16. van den Blink B, Juffermans NP, ten Hove T, et al. (2001) p38 mitogen-activated protein kinase inhibition increases cytokine release by macrophages in vitro and during infection in vivo. J. Immunol. 166: 582-587.

17. Sebolt-Leopold JS, Dudley DT, Herrera R, et al. (1999) Blockade of the MAP kinase pathway suppresses growth of colon tumors in vivo. Nat. Med. 5: 810-816.

18. Branger J, van Den Blink B, Weijer S, et al. (2002) Antiinflammatory effects of a p38 mitogen-activated protein kinase inhibitor during human endotoxemia. J. Immunol. 168: 4070-4077.

19. Hommes D, van den Blink B, Plasse T, et al. (2002) Inhibition of stress-activated MAP kinases induces clinical improvement in moderate to severe Crohn's disease. Gastroenterology 122: $7-14$.

20. Mizukami Y, Ura H, Obara T, et al. (2001) Requirement of cjun $\mathrm{N}$-terminal kinase for apoptotic cell death induced by farnesyltransferase inhibitor, farnesylamine, in human pancreatic cancer cells. Biochem. Biophys. Res. Commun. 288: 198-204.

21. Kolch W, Heidecker G, Kochs G, et al. (1993) Protein kinase C alpha activates RAF-1 by direct phosphorylation. Nature 364: 249-252.

22. Lebowitz PF, Davide JP, Prendergast GC. (1995) Evidence that farnesyltransferase inhibitors suppress Ras transformation by interfering with Rho activity. Mol. Cell. Biol. 15: 6613-6622. 